\title{
Increasing Safety And Reducing Environmental Damage Risk From Aging High-Level Radioactive Waste Tanks
}

Eric D. Steffler

Frank A. McClintock

W. Randolph Lloyd

Mark M. Rashid

Richard L. Williamson

Mili Selimotic

September 2005

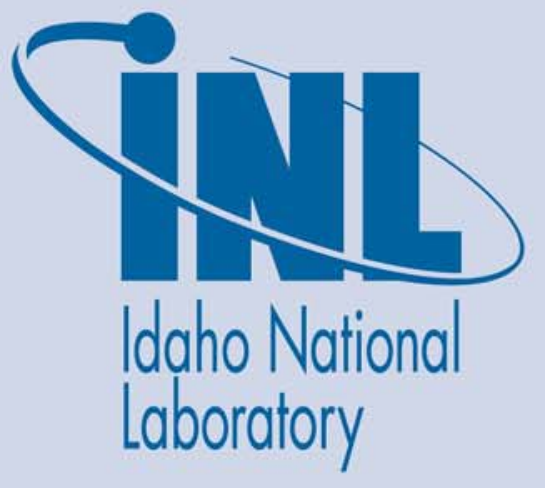

The INL is a U.S. Department of Energy National Laboratory operated by Battelle Energy Alliance 
Project Number: $\mathbf{8 1 8 9 8}$

Project Title: Increasing Safety and Reducing Environmental Damage Risk from Aging High-Level Radioactive Waste Tanks

Principal Investigator

Eric D. Steffler

Staff Scientist

Idaho National Laboratory

PO Box 1625, MS 2805

Idaho Falls, ID 83415

(208) 526-1074

(208) 526-4302 (fax)

eric.steffler@inl.gov

Co-Investigator

Frank A. McClintock

Professor Emeritus

Dept. of Mechanical Engineering

Massachusetts Institute of Technology

Cambridge, MA 02139

(978) 369-4098

(617) 258-8742 (fax)

fammwm@aol.com

Co-Investigator

W. Randolph Lloyd

Staff Scientist

Idaho National Laboratory

PO Box 1625, MS 2218

Idaho Falls, ID 83415

(208) 526-0808

(208) 526-4822 (fax)

randy.1loyd@inl.gov
Co-Investigator

Mark M. Rashid

Professor

University of California at Davis

Civil and Environmental Engineering

1 Shields Ave. 3123 Engineering III

Davis, CA 95616

(530) 752-7013

mmrashid@ucdavis.edu

Co-Investigator

Richard L. Williamson

Staff Scientist

Idaho National Laboratory

PO Box 1625, MS 2218

Idaho Falls, ID 83415

(208) 526-0576

(208) 526-4822 (fax)

richard.williamson@inl.gov

Doctoral Student Researcher

Mili Selimotic

Graduate Student

University of California at Davis

Civil and Environmental Engineering

1 Shields Ave. 3123 Engineering III

Davis, CA 95616

(530) 754-6420

mselimotic@ucdavis.edu 


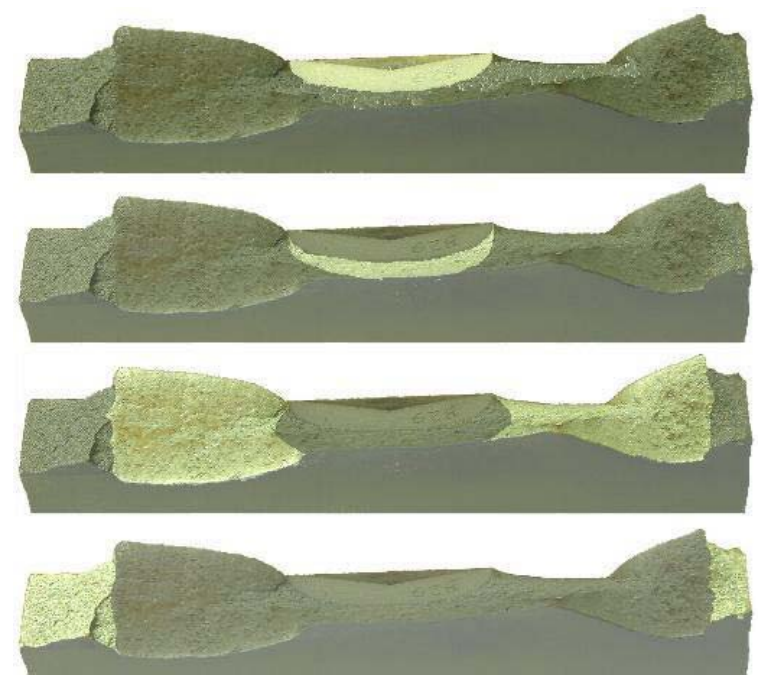

Figure 1. Stages of crack growth (highlighted): initial part-through surface crack (top); to penetration; then stable lateral growth; and finally, instability (bottom).

\section{EMSP Project 81898 - Increasing Safety and Reducing Environmental Damage Risk from Aging High-Level Radioactive Waste Tanks}

\section{Research Objective}

Cracks of various shapes and sizes exist in large high-level waste (HLW) tanks at several DOE sites. There is justifiable concern that these cracks could grow to become unstable causing a substantial release of liquid contaminants to the environment. Accurate prediction of crack growth behavior in the tanks, especially during accident scenarios, is not possible with existing analysis methodologies. This research project responds to this problem by developing an improved ability to predict crack growth in materialstructure combinations that are ductile (Fig. 1).

This new model not only addresses the problem for these tanks, but also has applicability to any crack in any ductile structure.

\section{Research Progress and Implications}

This report summarizes work progress through the fourth quarter of FY-05 (year 1 of a second 3-year funding period).

This research is developing a ductile crack growth prediction model, incorporating special capabilities [1] of the FEFRAC finite element code, and addresses several deficiencies in existing methodologies. The only methods currently available for assessment of crack growth in ductile materials have limitations. These methods include analytical/empirical approaches and numerical simulations. The analytical analyses, e.g. failure assessment diagrams (FAD), tend toward conservative estimates, and are limited to predicting either the onset of crack growth, or a safe/not-safe condition. The safe/not-safe condition provided by an FAD is not connected to a quantifiable structural performance measure, such as crack growth initiation or through-thickness penetration; in many cases, knowledge of these factors is critical. Conventional numerical models are restricted to pre-defined crack growth trajectories using node-release methods. Such models typically use pre-specified parameters, such as a fixed crack tip opening angle (CTOA), to control crack extension [2]. The new methodology, using FEFRAC as the computational platform, and crack extension controlled by a material separation function $[1,3]$, will provide a generalized crack growth prediction capability that includes potentially three-dimensional cracks within three-dimensional structures, and provides for completely autonomous crack growth trajectories. Materials of any type, including different materials within the same model, such as weld metal within surrounding base metal, can be specified. The model's unique method of predicting crack growth using the concept of a separation function allows modeling a broad range of crack growth mechanisms. FEFRAC uses a "patch mesh" that moves with the growing 


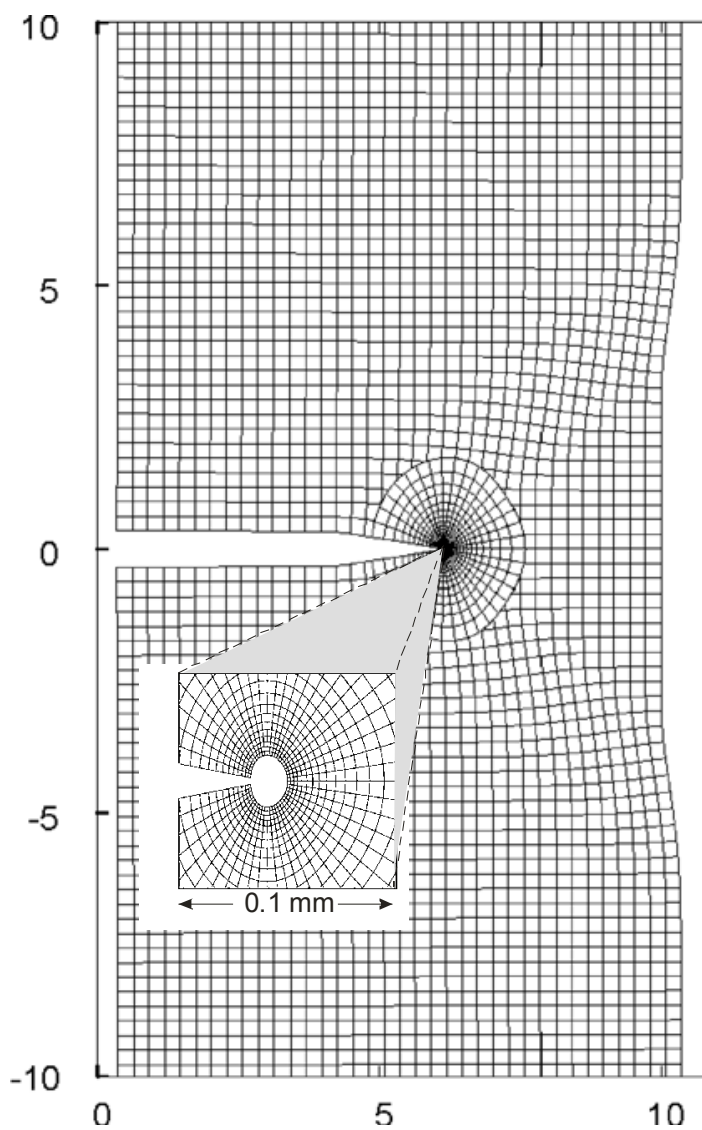

Figure 2. Deformed FEFRAC mesh from analysis of a single face-grooved extension specimen. The circular crack tip patch mesh travels with the moving crack tip. The small Exclusion Region [1] is located at the center of the patch and coincides with the instantaneous crack tip position (see inset). Dimensions are in $\mathrm{mm}$. crack tip (see Fig. 2). The separation function takes stress and/or displacement field-based parameters from the material response in the crack tip region at each model increment to control crack growth, rather than a fixed, pre-specified parameter.

The new model will provide accurate predictions for both onset of crack growth, through-thickness penetration, and crack growth stability analyses for the HLW tanks. Because of its generalized capabilities, the model will also be applicable to a broad range of ductile crack growth problems across the DOE complex and US industries. Assessment of crack growth resistance in standardized spent nuclear fuel storage canisters and other safety-significant structures built with ductile materials will be enhanced. This improved predictive capability will reduce the potential for severe environmental damage from catastrophic accidents, and serve to justify lifetime extension for critical structures when appropriate.

Significant advancements were made in several areas this year. Functionality and accuracy of the FEFRAC finite element model platform have been verified through comparisons with analytical solutions for non-hardening, rigid-perfectly plastic materials. FEFRAC model response using hardening material properties will be compared to experimental results in FY-06. FEFRAC predicts localized deformations associated with crack growth in perfectly plastic material with good accuracy and without numerical convergence problems. Various model performance issues were examined, and improvements were

implemented. Recent efforts have focused on fine-tuning performance of the two-dimensional models, and investigating localized or second order differences in model predictions compared to analytical solutions.

In further verification studies, slip-line-fracture-mechanics-type (SFLM) crack extension criteria have been implemented in a conventional finite element node-release crack growth formulation (ABAQUS). The displacement increment direction and stress normal to it were used to control crack extension by node release. The results for non-hardening material match the predicted theoretical behavior with acceptable accuracy.

Development of the numerical models so far has not required substantial experimental support. As generalized separation functions are developed for strain-hardening materials, experiments will be performed to assess their functionality and develop the necessary material coefficients. 
Experiments on single face-grooved extension specimens and part-through surface crack specimens made with strain-hardening materials confirm the localized bending that occurs early in the crack growth process, even when constrained, pure extension is applied.

Investigation of bending-type deformation (out-of-plane rotations) around part-through surface cracks or face cracks subjected to pure extension shows the phenomenon occurs with even small amounts of strain hardening. Strength-of-materials-type analysis shows the asymmetric geometry of the cracked cross-section creates off-center loading, causing plastic deformation away from the crack plane. Even constraint against bending applied close to the crack plane does not prevent this deformation from occurring. The resultant rotations of the crack flanks and surfaces near the crack mouth occur principally during the initial crack tip blunting. Rotation stops as the far-field stresses reach maximum values and the crack starts to grow. Crack growth then occurs under purely extensional deformation.

The computational work using FEFRAC and ABAQUS, as well as the idealized analytical solutions used to perform the verification studies, have been limited to planar geometries and plane-strain conditions. This setting is appropriate and useful for verification and for fracturecriterion development. However, crack extension in real structures clearly exhibits marked three-dimensional effects. This is particularly true for the part-through cracks in thin-walled tanks of special interest here. Accordingly, in connection with this project, work is well underway in extending the existing FEFRAC fracture-modeling capability to three dimensions. Key elements of this work include development of the three-dimensional ER "super element" and solid model variable element topology finite element method (VET-FEM) meshing and calculations [4]. A manuscript entitled "A Three-Dimensional Finite Element Method with Arbitrary Polyhedral Elements" describes features and performance testing of the VET-FEM and is in final stages of review for publication in the International Journal for Numerical Methods in Engineering.

\section{Planned Activities}

The co-investigators will meet at the INL in October to discuss and review recent research activities. A prioritized list of research activities for the coming year will be developed, along with the related schedule.

Two manuscripts discussing the FY-05 research and results related to embedding separation functions motivated by SLFM concepts into finite element models for ductile crack growth are in final preparation, to be submitted in December 2005. One, presented at the International Symposium on Plasticity 2005, will be submitted to the International Journal of Plasticity. The other manuscript will go to Engineering Fracture Mechanics for consideration.

a The Exclusion Region, or ER, is not an element in the conventional finite element sense, but rather is a region in the model where enhanced, or special, constitutive relations are applied. These relationships allow for discontinuous displacement within the region, making the ER especially useful for crack growth modeling. See reference 1 for details on ER theory. 
Work by Professor Rashid and Mr. Selimotic will continue on the three-dimensional implementation of FEFRAC, including the ER super-element and associated separation functions, and the solid element VET remeshing algorithm.

Experiments in the first half of FY-06 on different materials and specimens will test some of the responses predicted by the developing methods. Newly designed tests will quantify the localized bending deformation. Following the physical testing, the INL microtopography system [5] will analyze the broken specimens. These results provide three-dimensional crack opening and crack extension history for the tests - important data that cannot be obtained during the test.

The test results from various materials and specimen geometries will provide calibration information for the FEFRAC model, such as the crack mouth opening versus applied force relationships. The microtopographic data will provide for a direct comparison of the kinematic behavior, e.g. crack tip blunting, crack growth rates, and crack trajectories predicted by the FEFRAC model. The combined data will be used to refine the separation function(s), with the goal of rendering reliable, accurate, and autonomous predictions of ductile crack growth in real three-dimensional structures.

\section{References}

1. Rashid, M.M. and V. Tvergaard (2003), "On the path of a crack near a graded interface under large scale yielding,” Int. J. Solids and Structures 40, pp. 2819-2831.

2. Newman Jr., J. C., M. A. James, and U. Zerbst (2003), "A Review of the CTOA/CTOD Fracture Criterion,” Eng. Frac. Mech. 70, pp. 371-385.

3. Rashid, M.M. and V. Tvergaard (2004), "Effect Of A Graded Interface On A Crack Approaching At An Oblique Angle,” Int. J. Comp. Eng. Sc. 5(4), pp.781-794.

4. Rashid, M. M. (2002), “Material State Remapping in Computational Solid Mechanics,” Int. J. Numerical Methods Eng. 5, pp. 431-450.

5. Lloyd, W. (2003), "Microtopography for Ductile Fracture Process Characterization-Part 1: Theory and Methodology." Eng.Fracture Mechanics 70, pp. 387-401. 